\title{
On Angle References in Long-Term Time-Domain Simulations
}

\author{
Davide Fabozzi, Student Member, IEEE, and Thierry Van Cutsem, Fellow, IEEE
}

\begin{abstract}
In power system dynamic models, the complex network equations and the various phasors are projected onto reference axes. After a short critical review of commonly chosen reference axes, this letter proposes to use the center-of-inertia at the previous integration time step. This approach is shown to combine the advantages of the center-of-inertia with a sparser Jacobian structure and an easier handling of network splits.
\end{abstract}

Index Terms-Angle reference, long-term dynamics, network equations, phasor approximation, time-domain simulation.

\section{STANDARD ANGLE REFERENCES}

$\mathbf{T}$ HE dynamic model of a power system considered in stability studies can be written in compact form as [1]

$$
\begin{aligned}
0 & =g(x, y) \\
\dot{x} & =f(x, y) .
\end{aligned}
$$

Equations (1) relate to the network, whose response is assumed instantaneous under the phasor approximation. They are usually derived from the $\mathbf{Y}$ bus admittance matrix relations $\mathbf{Y} \overline{\mathbf{V}}=\overline{\mathbf{I}}$, where $\overline{\mathbf{V}}$ (resp. $\overline{\mathbf{I}}$ ) is the vector of bus voltage (resp. current) phasors.

It is appropriate to project these complex equations onto two orthogonal axes, denoted $x$ and $y$, respectively. Let $v_{x i}$ and $v_{y i}$ denote the projections of the bus voltage $\bar{V}_{i}$ onto the above axes. The components relative to the various buses are grouped into $\mathbf{v}_{x}$ and $\mathbf{v}_{y}$ vectors, which are included in $\mathbf{y}$. The current vectors $\mathbf{i}_{x}$ and $\mathbf{i}_{y}$ are defined similarly.

Equations (2) deal with a variety of short- and long-term phenomena and controls, with corresponding state variables in $\mathbf{x}$. Among them, the motion of the rotor of the $i$ th synchronous machine $(i=1, \ldots, m)$ is described by

$$
\begin{aligned}
\dot{\theta}_{i} & =\omega_{i} \\
M_{i} \dot{\omega}_{i} & =T_{m i}-T_{e i}
\end{aligned}
$$

where $M_{i}$ is an inertia coefficient, $T_{m i}$ (resp. $T_{e i}$ ) is the turbine mechanical (resp. generator electromagnetic) torque, $\omega_{i}$ is the rotor speed, and $\theta_{i}$ the rotor position with respect to a fixed reference.

Apart from the need to have all phase angles referring to the same axis, the choice of the latter is free. In fact, all variables in $\mathbf{x}$ except angles are unaffected by this choice. The $x$ and $y$ axes should be chosen having in mind computational efficiency. A

Manuscript received July 23, 2009; revised October 23, 2009. First published March 11, 2010; current version published January 21, 2011. This work was performed in the context of the PEGASE project funded by European Community's 7th Framework Programme (grant agreement No. 211407). Paper no. PESL-00086-2009.

D. Fabozzi is with the Department of Electrical Engineering and Computer Science (Montefiore Institute) of the University of Liège, B-4000 Liège, Belgium.

T. Van Cutsem is with the Fund for Scientific Research (FNRS), University of Liège, B-4000 Liège, Belgium (e-mail: t.vancutsem@ulg.ac.be).

Digital Object Identifier 10.1109/TPWRS.2010.2042652 standard practice is to choose axes that rotate at the nominal angular frequency $\Omega_{o}=2 \pi f_{o}$, where $f_{o}$ is the nominal frequency of the system. The rotor angle of the $i$ th machine, referred to the $x$-axis, is

$$
\delta_{i}=\theta_{i}-\left(\omega_{o} t+C\right)
$$

where $C$ is an arbitrary constant. This yields

$$
\dot{\delta}_{i}=\omega_{i}-\omega_{o}
$$

This equation is substituted to (3), while (4) is unchanged.

While appropriate for short-term simulations (e.g., for transient stability analysis), the above reference suffers from a major drawback in long-term studies. Indeed, after a disturbance affecting the power balance, the system settles at a new angular frequency $\Omega$ and, with respect to the reference axes, all phasors rotate at the angular speed $\Omega-\Omega_{o}$. Hence, although the system settles at a new equilibrium, the $\delta_{i}$ variable increase linearly with time and the components of $\mathbf{v}_{x}, \mathbf{v}_{y}, \mathbf{i}_{x}$ and $\mathbf{i}_{y}$ oscillate with a period $T=2 \pi\left|\Omega-\Omega_{o}\right|^{-1}$. This nonlinear behavior requires frequent updates of the Jacobian matrix and increases the number of iterations of the Newton method used to solve the implicit equations [1]. It also requires the time step to remain small compared to $T$, in order to track those oscillations and/or avoid numerical instability.

The above problem can be solved by somewhat linking the reference axes to the rotor motion. This is the idea underlying the center-of-inertia (COI) reference frame, which has been used in direct transient stability analysis methods [2] and in industrial simulation software (e.g., [3]). The COI angle and its time derivative are defined, respectively, as

$$
\begin{aligned}
& \theta_{c o i}=\frac{1}{M_{T}} \sum_{i=1}^{m} M_{i} \theta_{i}+K \\
& \omega_{c o i}=\frac{1}{M_{T}} \sum_{i=1}^{m} M_{i} \omega_{i}
\end{aligned}
$$

where $M_{T}=\sum_{i=1}^{m} M_{i}$ is the total inertia and $K$ is an arbitrary constant. The $x$ and $y$ axes now turn at the angular speed $\omega_{c o i}$. The rotor angle of the $i$ th machine, referred to the $x$-axis, is

$$
\delta_{i}=\theta_{i}-\theta_{c o i}
$$

from which one easily derives

$$
\dot{\delta}_{i}=\dot{\theta}_{i}-\dot{\theta}_{c o i}=\omega_{i}-\omega_{c o i} .
$$

This equation replaces (3). Thus, in an $m$-machine system, there are $m$ of the type (10) involving $m$ state variables $\delta_{i}$ and the $(m+1)$ th variable $\omega_{c o i}$. The latter is included in $\mathbf{y}$ and is balanced by (8), an algebraic equation handled by the Newton method together with (1). Note that (8) can be replaced by $\sum_{i=1}^{m} M_{i} \delta_{i}=0$. 
Clearly, if the system settles at a new equilibrium with frequency $f$, all machines rotate at the angular speed $2 \pi f$ and so does the $x$ axis. Hence, $\mathbf{v}_{x}, \mathbf{v}_{y}, \mathbf{i}_{x}$ and $\mathbf{i}_{y}$ become constant.

This result still holds true when substituting to the right-hand side of (8) a linear combination $\sum_{i=1}^{m} a_{i} \omega_{i}$ with $\sum_{i=1}^{m} a_{i}=$ 1 , and in particular a single reference speed. The COI frame of reference, however, is appealing as it provides the average system angular frequency. The latter is appropriately used in frequency-dependant load models as well as in corrective damping terms $D_{i}\left(\omega_{i}-\omega_{c o i}\right)$ added to (4).

Still, this COI reference suffers from drawbacks. First, the presence of $\omega_{c o i}$ in each (10) and in the load models produces a dense column in the Jacobian matrix, while the presence of all $\omega_{i}$ 's in (8) produces a "dense" row. Next, in case of network split, since one COI has to be considered in each connected subnetwork, the structure of the Jacobian is significantly modified by the added variables and equations. Finally, the algebraic equation (8) does not allow to take advantage of the block bordered diagonal structure of the Jacobian (see, e.g., [1, pp. 794-795]).

The approach described below is free from these drawbacks.

\section{Proposed Angle Reference}

The idea of the proposed technique is to make the $x$ axis coincide with a previous position of the COI. In the context of numerical simulation, when integrating the equations from $t-h$ to $t$ (where $h$ is the time step), the phasors are projected onto axes that correspond to the COI position at $t-h$. At that time, the COI speed is $\omega_{c o i}(t-h)$. The angle and speed of the $i$ th machine, referred to that axis, become

$$
\begin{aligned}
& \delta_{i}=\theta_{i}-\theta_{c o i}(t-h) \\
& \dot{\delta}_{i}=\omega_{i}-\omega_{c o i}(t-h) .
\end{aligned}
$$

Note that the COI considered in (10) is "implicit" in the sense that it involves the rotor speeds at the same time $t$, while the one considered in (12) is "explicit" in so far as it refers to past, already known speed values. In (12), $\omega_{c o i}(t-h)$ is just a number, not a variable. Equation (8) is not handled together with the other equations. It is used, after convergence of the Newton iterations, to determine the value of $\omega_{\text {coi }}(t)$ from the just computed rotor speeds $\omega_{i}(t)$. This updated value of $\omega_{c o i}$ is used in (12) when proceeding with the next integration step.

By so doing, the dense row and column are no longer present in the Jacobian, while network splits do not require any restructuring of the Jacobian.

The advantages of the COI reference frame are also preserved, because a slightly delayed COI angle is as good as the exact COI in making $\mathbf{v}_{x}, \mathbf{v}_{y}, \mathbf{i}_{x}$, and $\mathbf{i}_{y}$ very little dependent on the system frequency. One could argue that using a slightly delayed $\omega_{c o i}$ in the frequency dependent load models causes some inaccuracy; however, using $\omega_{c o i}$ instead of the true angular frequency at the load bus already involves some approximation.

\section{ILLUSTRATIVE EXAMPLE}

We consider a variant of the system used in [4], subject to a branch outage. All responses have been computed using the Trapezoidal method with $h=0.01 \mathrm{~s}$.

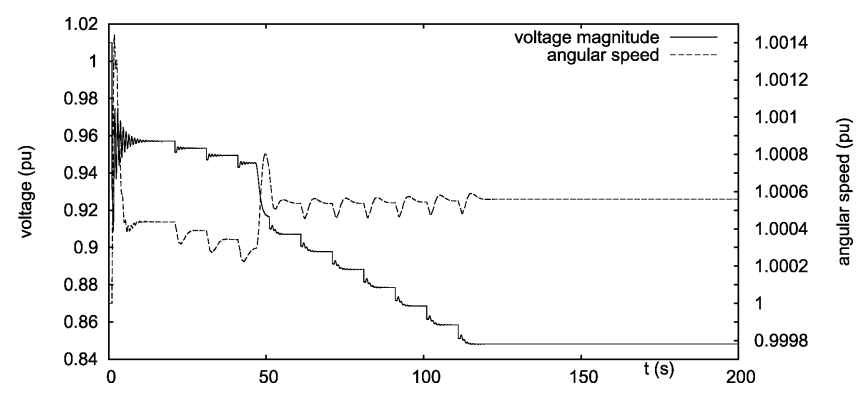

Fig. 1. Evolution of voltage and COI angular speed.

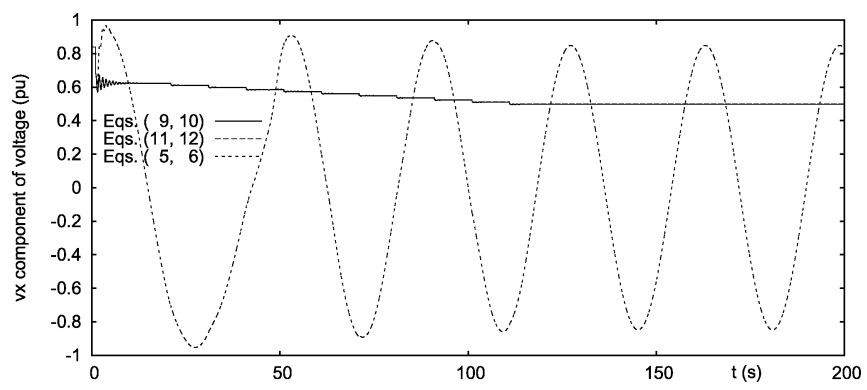

Fig. 2. Evolution of a $v_{x}$ component.

The long-term evolution of a bus voltage magnitude is shown with solid line in Fig. 1. The system responds to an overexcitation limiter acting at $t \simeq 50 \mathrm{~s}$ and a transformer load tap changer moving by 10 steps, before hitting its limit. The evolution of $\omega_{c o i}$ is shown with dotted line in the same figure. The overall increase is due to the load sensitivity to the dropping voltage. As can be seen, the system settles at equilibrium after $t \simeq 120 \mathrm{~s}$.

Apart from numerical inaccuracies (within the solver tolerances), $\omega_{c o i}$ is the same, whichever angle reference is used, since the $\omega_{i}$ 's are also the same, as stated in Section I.

Fig. 2 shows with dotted line the evolution of the $v_{x}$ component of the same bus voltage, when using the angle reference as in (5). As expected, $v_{x}$ undergoes pronounced oscillations, even after the frequency has returned to steady state. The other two curves have been obtained with the angle references defined by (10) and (12), respectively. The corresponding reference axes differ by the angle $\int_{t-h}^{t} \omega_{c o i}(u) d u=\theta_{c o i}(t)-\theta_{c o i}(t-h)$, which is small for $h=0.01 \mathrm{~s}$, and hence makes the curves indistinguishable. More importantly, these two curves are much smoother than the first one.

\section{CONCLUSION}

The proposed, explicit COI reference frame yields smoothly varying voltage components, appropriate for long-term simulation, while preserving the sparsity of the Jacobian and offering an easy handling of network splits.

\section{REFERENCES}

[1] P. Kundur, Power System Stability and Control, ser. EPRI Power System Engineering. New York: McGraw-Hill, 1994.

[2] C. J. Tavora and O. J. M. Smith, "Characterization of equilibrium and stability in power systems," IEEE Trans. Power App. Syst., vol. PAS-91, pp. 1127-1130, 1972.

[3] Eurostag Package Release 4.4, User's Manual, Part 1.

[4] D. Fabozzi and T. V. Cutsem, "Simplified time-domain simulation of detailed long-term dynamic models," in Proc. IEEE PES General Meeting, Calgary, AB, Canada, Jul., 2009, paper No. 09GM1061. 